\title{
THE EFFECT OF CERVICAL DORSAL ROOT AND SYMPATHETIC STIMULATIONS ON THE VESTIBULORADIAL RESPONSE IN THE CATS
}

\author{
Kazuo OKada, Toru Matsunaga*, Setsuo Arai, \\ Naosaburo YosHII and Akira ADACHI
}

Head and neck injuries caused by whiplash during traffic accidents frequently result in vertigo. It is characterized by an absence of organic change in the vestibulum. Many etiological factors have been proposed as causes of this vertigo, such as bleeding in the cervical spinal cord, disturbance of circulation in the internal ear, hypertonicity of the cervical sympathetic nerves, etc. Most of the hypothesis, however, lack of critical evidence, possibly due to technical difficulties in the performance of experiments.

It seems reasonable to assume that some functional disorder caused by whiplash injury would be detectable in the vestibulum or in its functionally related structures, even though organic change cannot be found there. Certainly, one factor rather alone among the many which have been proposed the vertigo; many factors together would be involved in this sequelae.

The motoneuron response evoked by vestibular stimulation has been electrophysiologically analyzed in full detail by Gernandt \& Gilman. ${ }^{11}$ It is profitable to apply these results for the understanding of vestibular functions. Thus, the present experiment was designed to clarify the mechanism producing vertigo caused by whiplash injury, with emphasis on the functional relationship between the vestibulospinal motor system and the cervical sympathetic as well as the cervical somatic afferent systems.

\section{METHOD}

The experiments were carried out on adult cats. They were mounted on a stereotaxic apparatus under ether anesthesia and the trachea was canulated. The animals were immobilized by Flaxidil and maintained on artificial respiration. Stimulating monopolar electrode was stereotaxically inserted into Deiters nucleus, and the indifferent electrode was placed on the temporal muscle.

The cervical sympathetic nerve was exposed and transected between the superior and medial cervical ganglia. Bipolar stimulating electrodes were placed on the inferior cut end. The other electrodes were also placed on the cervical dorsal root for stimulation. Two electronic stimulators with isolator units were used. One stimulator which provides a rectangular pulse of suprathreshold intensity and of fixed duration within the range of 0.5 to $0.7 \mathrm{msec}$. was connected for Deiters nucleus stimulation. The stimulation was *the Department of Oto-Rhino-Laryngology, Osaka University, Medical School, Osaka 

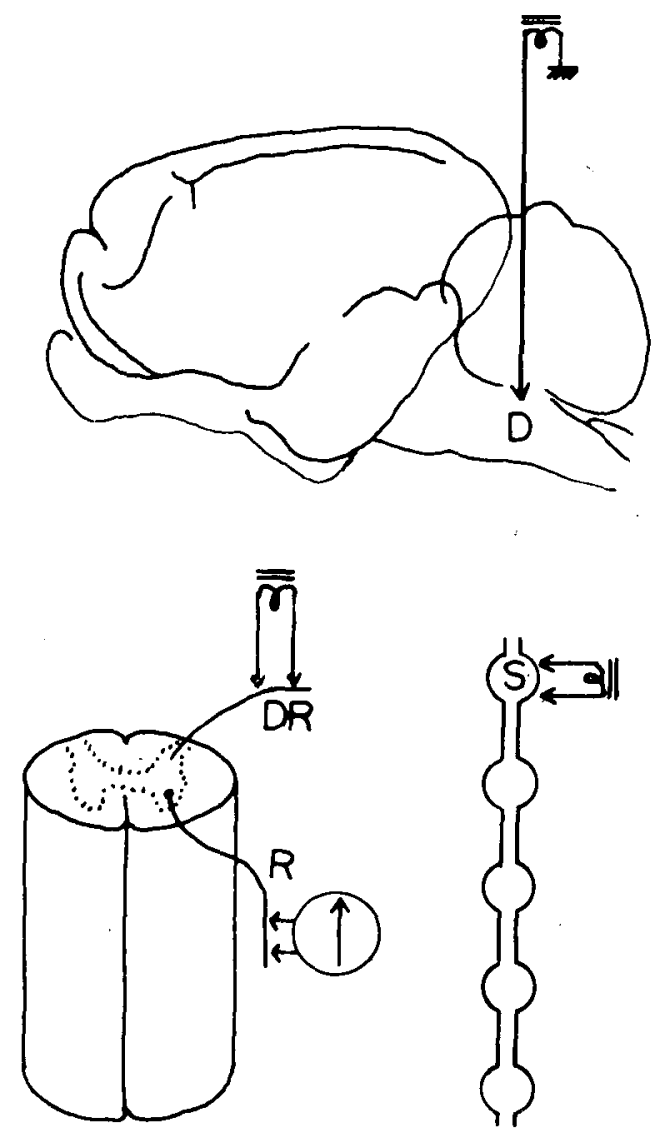

Fig 1. Schematic diagram of experimental procedure. Symbols. D : Deiters nucleus. DR: Dorsal root. S: Sympathetic nerve. R: Radial nerve

ordinarily delivered at a rate of 0.5 per second. In order to examine the effect of sympathetic or somatic afferent stimulation the other stimulator provided repetitive pulses of 20 to 30 per second, with intensity of 3 to $5 \mathrm{~V}$ to the ipsilateral cervical sympathetic nerve or dorsal root. When recovery rates were measured, Deiters nucleus was stimulated by a pair of rectangular pulses with controlled interrelated delays and of the same intensity. The recovery rates obtained in this way were compared with those obtained during sympathetic stimulation by means of another electronic stimulator which provided repetitive pulses of subthreshold intensity.

The radial nerve was also exposed and dissected into filaments. A filament was placed on a bipolar recording electrode. Both the recording and stimulating electrodes were made of stainless steel. The exposed nerve filament was covered with paraffin oil immediately after it was mounted on the electrode to avoid drying. Amplification was obtained by using differential AC-coupled amplifiers leading into an oscilloscope. Photographs were made on film by a kymograph camera. The experimental procedure was schematically shown in Fig. 1. 


\section{RESULTS}

I. Radial motoneuron response to vestibular and cervical sympathetic stimulations.

A motoneuron response to single shock stimulation of Deiters nucleus was recorded from the ipsilateral radial nerve, as shown in Fig. 2A. The response consists of an initial spike which appears after 2 to $3 \mathrm{msec}$. latency followed by late activities with longer duration of approximately $10 \mathrm{msec}$. This is quite similar to the vestibular response described by Gernandt and Gilman. ${ }^{11}$ It should be noted that no difference in shape or amplitude of the response evoked by each single shock stimulation was recognized as long as the single shock was delivered at intervals longer than $1 \mathrm{sec}$. Fig. $2 \mathrm{~B}$ shows a response of the radial nerve to single shock stimulation of the ipsilateral cervical sympathetic nerve. This response displays a complicated shape. Several negative peaks are always observed. The latency from the onset of stimulation to the maximal peak is approximately $15 \mathrm{msec}$. Amplitude of the response was attenuated by repetitive stimulation of more than $0.5 \mathrm{c} / \mathrm{sec}$. It suggests that this response would be mediated by polysynaptic reflex arc.

II. Sympathetic effect on vestibulo-radial response.

It should be interesting to examine the sympathetic influence on the descending vestibular response. Fig. 3 presents behavior of the vestibular response during repetitive stimulation of the ipsilateral cervical sympathetic nerve. The late activities of the vestibular response recorded from the radial nerve were attenuated when the sympathetic nerve was stimulated by repetitive pulses of 30 per second with intensity of $7 \mathrm{~V}$ and $0.5 \mathrm{msec}$. dura-

A
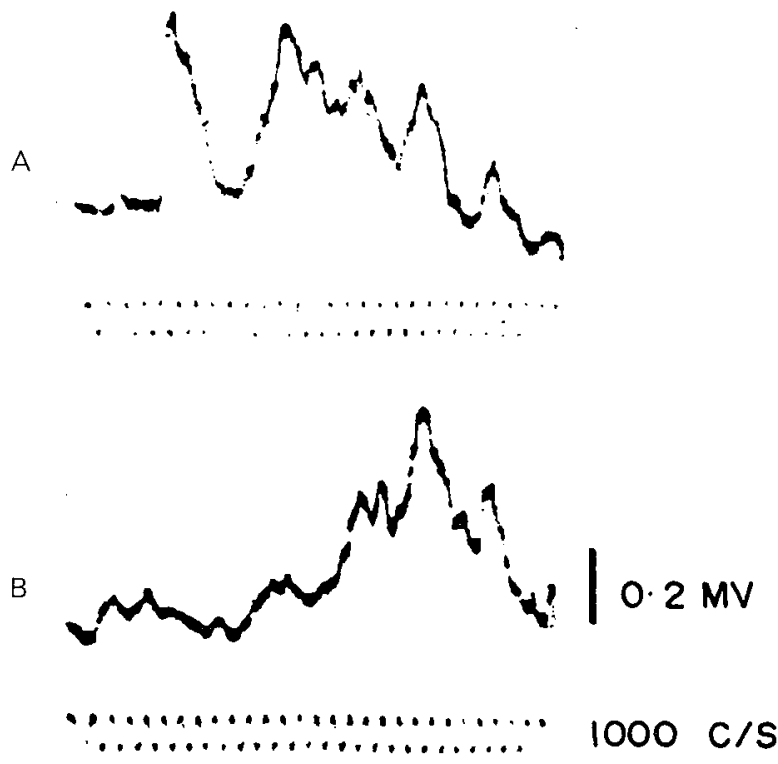

Fig. 2. Vestibular response ( $5 \mathrm{~V} .0 .7 \mathrm{msec}$ ) recorded from the ipsilateral radial nerve in $A$. Response elicited by a single shock stimulus of the ipsilateral cervical sympathetic nerve, recorded from the radial nerve in $B$. 

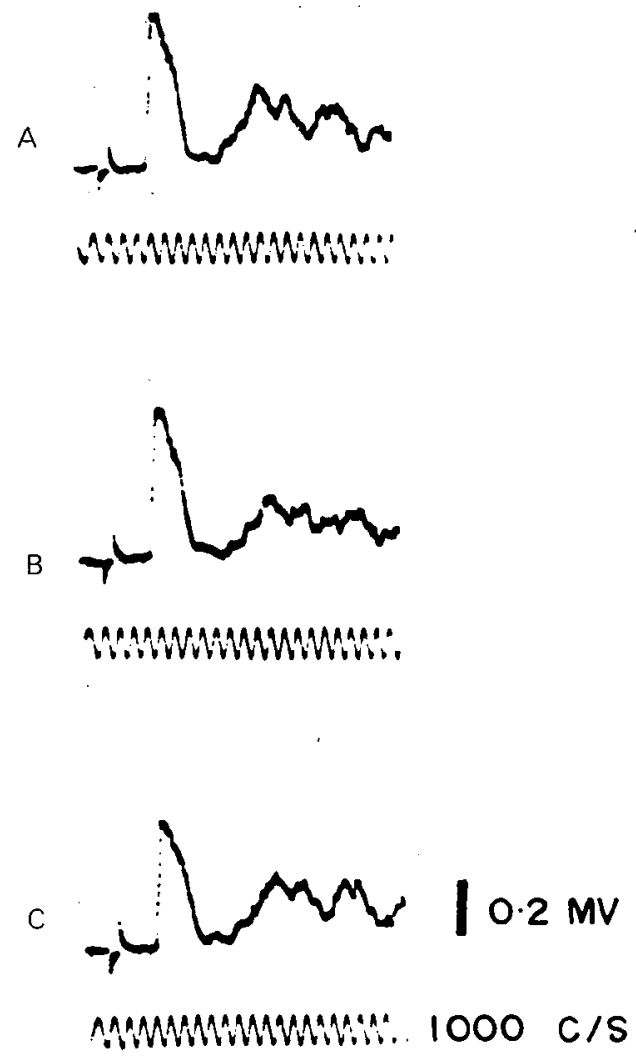

Fig. 3. A: Evoked potentials were recorded from the ipsilateral radial nerve when a single shock ( $6 \mathrm{~V}, 0.5 \mathrm{msec}$.) was applied to the vestibular nucleus. B: Inhibitory effect on the late activities during repetitive stimulation ( $5 \mathrm{~V}, 0.2 \mathrm{msec} ., 20 \mathrm{c} / \mathrm{s}$ ) of the ipsilateral cervical sympathetic nerve. $C$ : Recovery of the late activities immediately after cessation of the sympathetic stimulation.

tion, as shown in Fig. 3B. This inhibition completely recovered immediately after the cessation of sympathetic stimulation. However, no effect was recognized on the initial spike. It revealed that cervical sympathetic nerve stimulation can modulate the motoneurone response of the radial nerve elicited by vestibular stimulation. This fact suggests that mechanical as well as electrical stimulation might inhibit the vestibulo-radial motor response.

III. Effect of section or mechanical stimulation of the sympathetic nerve on vestibuloradial response.

To prove this suggestion the next experiment was carried out. Ascertaining that almost the same response (cf. Fig. 4A) as presented above was elicited by vestibular stimulation, the ipsilateral sympathetic nerve was transected between the superior and medial ganglia. Fig. 4B shows the vestibular response immediately after the transection. Amplitude of the late activity was markedly inhibited. This inhibition disappeared $2 \mathrm{~min}$. 


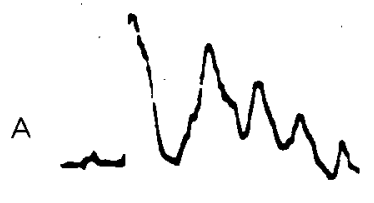

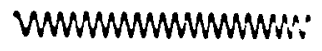
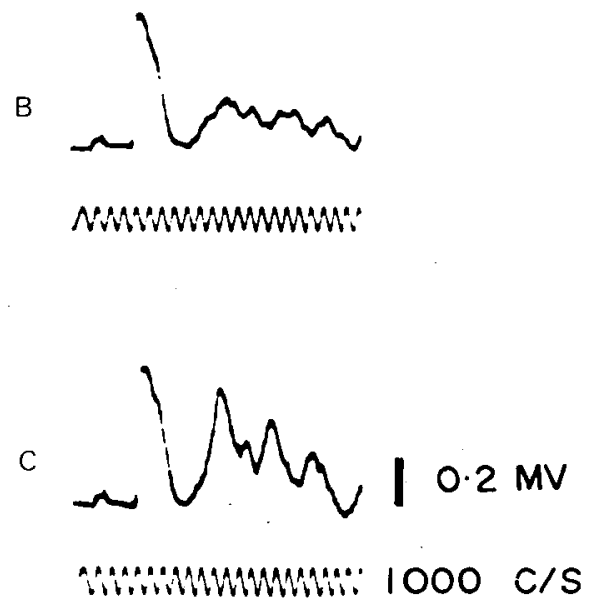

Fig. 4. Inhibitory effect of the ipsilateral cervical sympathetic transection on the late activities in the vestibulo-radial pathways. A : Control response before the transection. B : Response obtained immediately after the transection. $\mathrm{C}$ : Response recorded $2 \mathrm{~min}$. later.

after the tarnsection as shown in Fig. 4C. After recovery the inhibitory effect on the late activities was seen when an inferior cut end of the sympathetic nerve was stimulated electrically.

IV. Effect of dorsal root stimulation on vestibulo-radial response.

Fig. 5 shows the effect of the ipsilateral cervical dorsal root stimulation on the vestibulo-radial response. In this case, the dorsal surface of the spinal cord was exposed and after transecting the dorsal root, a central end was stimulatd. This surgery always caused depression of the initial spike amplitude and enhancement of the late activities. Fig. 5A shows the response obtained after transection of the dorsal root. The late activity was almost completely diminished in size during repetitive stimulation of the dorsal root with 30 pulses per second, $0.2 \mathrm{msec}$. duration (Fig. 5B). As the intensity of the stimulation was selected so as not to initiate any reflex, no response was elicited in the radial nerve by the recovery from inhibition after the cessation of stimulation.

V. Effect of sympathetic nerve stimulation on recovery curve of vestibulo-radial response.

Recovery rates were measured in order to analyse further the effect of modulating stimuli such as sympathetic stimulation on the vestibulo-radial response. (Fig. $6 \mathrm{~A}, \mathrm{~B}$ ) 

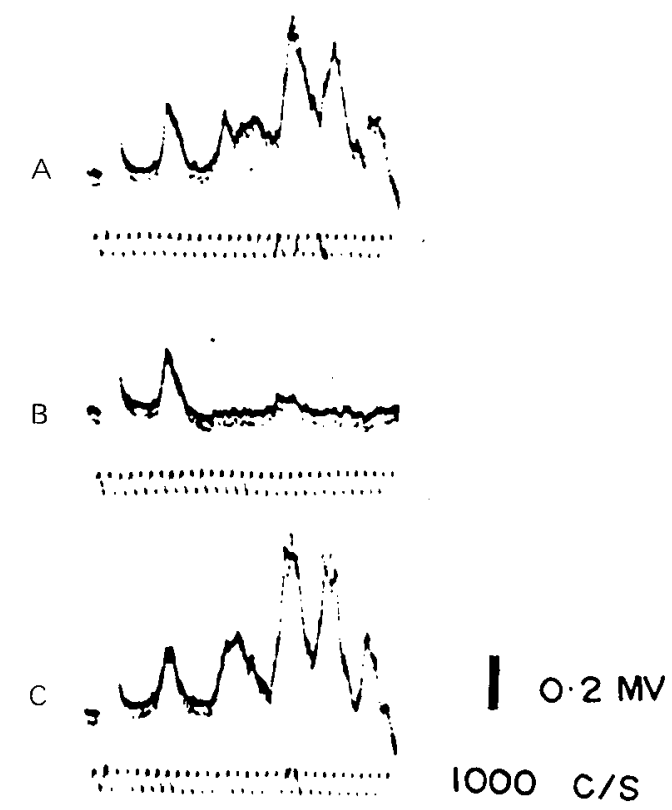

Fig. 5. A: Control response. B: Complete inhibition of the late activities during repetitive stimulation ( $3 \mathrm{~V}, 0.2 \mathrm{msec} ., 30 \mathrm{c} / \mathrm{s})$ of the $C_{1}$ dorsal root. $C$ : Recovery after cessation of the dorsal root stimulation.

Two successive shocks were applied to the vestibular nucleus and the responses to each shock were recorded from the radial nerves. The interval of the two shocks was progressively widened from $20 \mathrm{msec}$. to $1000 \mathrm{msec}$., because the ongoing response induced by the initial conditioning shock prohibited correct measurement of amplitude of the response which was evoked by a second test shock with the interval less than $20 \mathrm{msec}$. It provides another way to evaluate the effect of sympathetic stimulation. When the two shocks were delivered in rapid succession of $20 \mathrm{msec}$. to $150 \mathrm{msec}$., the amplitude of the initial spike was decreased (subnormal period). The recovery rates were increased again with the intervals more than $150 \mathrm{msec}$. The second enhancement in this period was less than that in the initial super-normal period. This recovery rate of the vestibulo-radial response was compared with that during sympathetic stimulation. The two curves show similar enhancement during the super-normal period. However, a difference was recognized in the subnormal and the second enhancement period. During those inter-stimuli intervals more than $50 \mathrm{msec}$, the recovery rates of the initial spikes were significantly suppressed during sympathetic stimulation (Fig. 6A). The result indicates that initial spikes which seemed to be stable in former experiments are also modifiable by sympathetic stimulation.

The same experiment was repeated on the late activity of the vestibulo-radial response. Since the late activity always displayed several peaks, amplitude was measured on the highest peak. In this case, interrelated intervals between two shocks were progressively widened from 10 msec., because the late activity was elicited after longer latency than the initial spike. The recovery rate of the late activity was different from that of the initial spike 


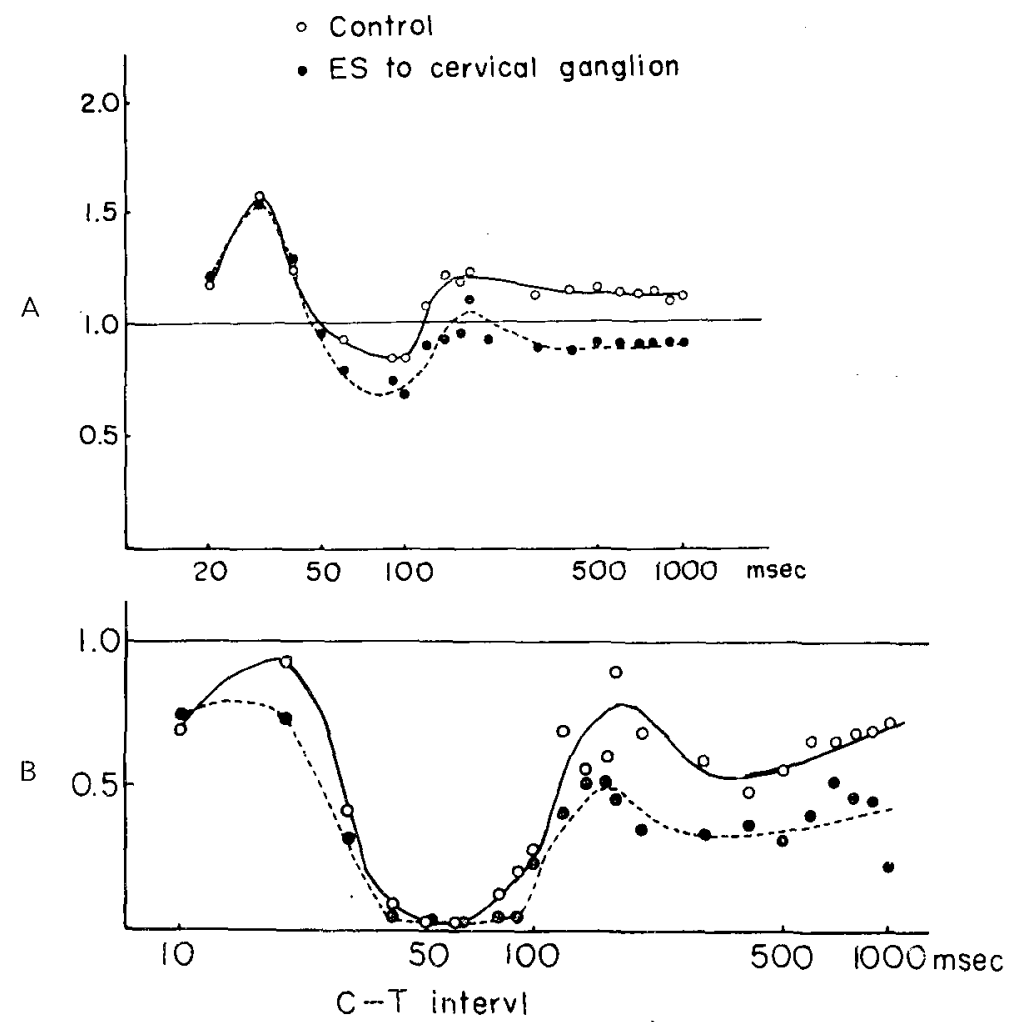

Fig. 6. A: Comparison of recovery curves of the initial spike in the vestibuloradial response during sympathetic stimulation with the control recovery curve obtained during no sympathetic stimulation in the cerebellum intact animal. Ordinate: Recovery rate. Abscissa: Interval in insec. between conditioning and test stimuli. Open circles: Recovery rates obtained without sympathetic stimulation (control). Solid circles: Recovery rates obtained during cervical sympathetic stimulation (10V. 0.3 msec., $30 \mathrm{c} / \mathrm{s}$ ). B: Comparison of recovery curve of the late activity (highest peak) in the vestibulo-radial response illustrated by the similar way in $A$.

as presented in Fig. 6B. The recovery rate was approximately 0.7 at $10 \mathrm{msec}$. interval. It reaches almost 1.0 at $20 \mathrm{msec}$. However, it declined rapidly after that, without the supernormal period which was markedly recognized on the initial spike. The late activity induced by the test shock almost disappeared during the inter-stimuli interval of 50 to $60 \mathrm{msec}$. After the complete inhibition, the recovery rate increases again, but no complete recovery was recognized even at $1000 \mathrm{msec}$. interval. The recovery of the late activity formed a large waving curve when plotted against time. It was characterized by a complete absence of the super-normal period. The recovery rate obtained during the sympathetic stimulation presented lower values at all conditioning-test stimuli intervals except at the interval of 50 to $60 \mathrm{msec}$. when the control recovery rates showed zero.

VI. Role of the cerebellum.

To analyse the role of the cerebellum in the effects of sympathetic stimulation, the same experiments were carried out on decerebellated animals. Fig. 7A shows the recovery 

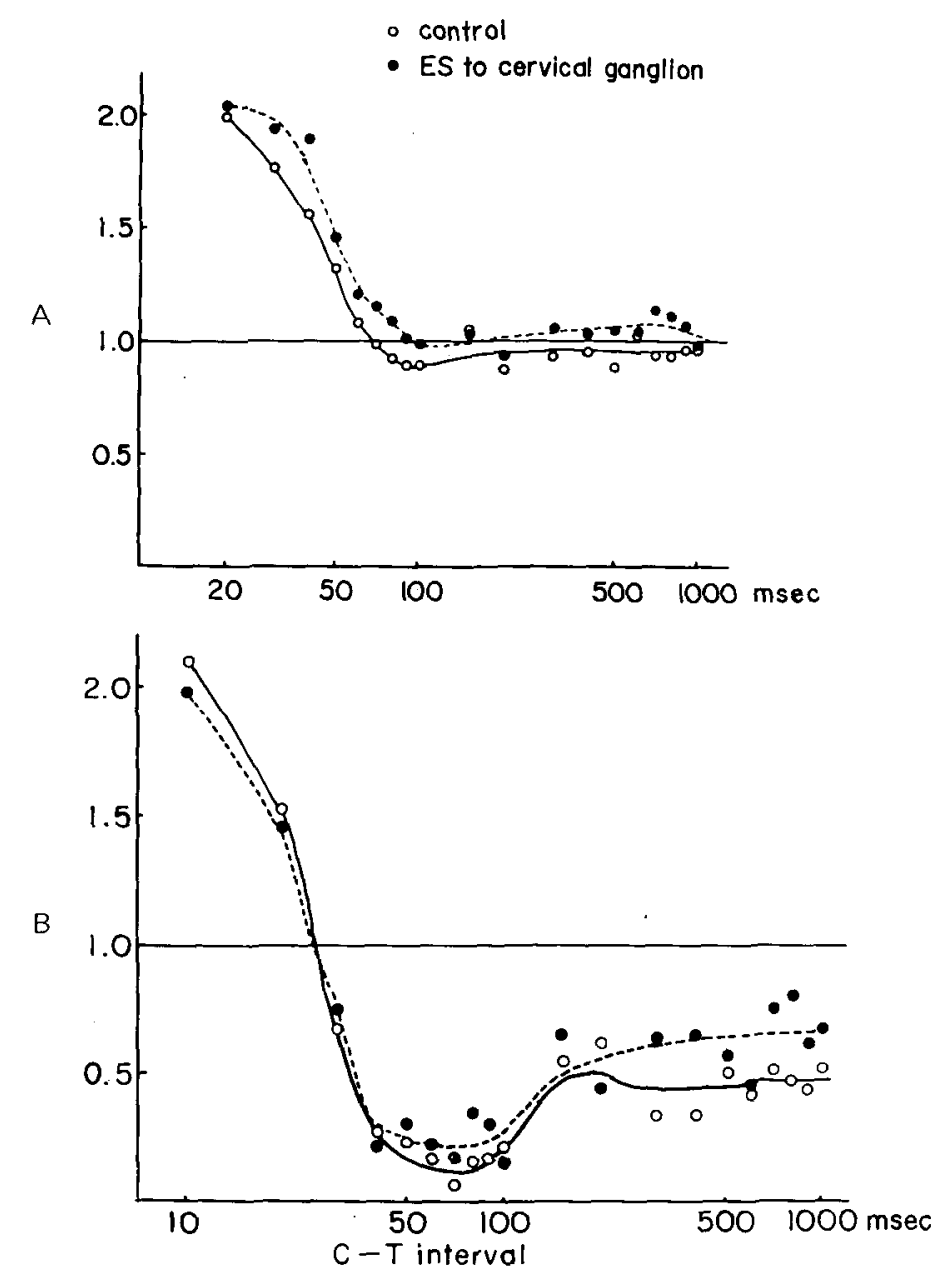

Fig. 7. A: Effect of cerebellectomy on both recovery rates of the initial spikes as presented in Fig. 6. B: Effect of cerebellectomy on both recovery rates of the late activities.

curve of the initial spike obtained from the decerebellated cat. It is noted that the intial recovery rate at the intervals of $20 \mathrm{msec}$. was markedly facilitated in the decerebellated preparation, when the recovery curves are compared with that in Fig. 6A. The subnormal period and the second enhancement period were not obesrved. Moreover, the recovery curve during sympathetic stimulation was the same as that without stimulation but with slightly greater values at all intervals. Fig. 7B shows the same comparison of recovery curves measured at the highest peak of the late activity. In the late activity the enhancement of the recovery rate was observed in the decerebellated animal at less than $25 \mathrm{msec}$. intervals. No difference between two curves was noted at the intervals less than $100 \mathrm{msec}$., while the facilitation of the recovery rates was observed during sympathetic stimulation in the intervals more than $300 \mathrm{msec}$.

The effects of decerebellation on both recovery rates of the initial spike and the late 
activity were summarized as follows: an increment of recovery rate in the short intervals of less than $20 \mathrm{msec}$. and the reversal of inhibitory effect of the recovery curves to their facilitation at the intervals more than $300 \mathrm{msec}$. during sympathetic stimulation in the decerebellated preparation. It is suggested that the cerebellum plays some inhibitory role in the vestibulo-radial response and that sympathetic inhibition of the vestibulo-radial response is much influenced by cerebellar tonus.

\section{DISCUSSION}

The vestibular responses recorded from the ipsilateral radial nerve in the present experiment resemble those reported by Gernandt, Katsuki \& Livingston ${ }^{9}$; Gernandt \& Gilman, ${ }^{11}$ in that they consist of a negative spike (the initial spike) followed by poorly synchronized smaller negative waves (the late activity). Dissimilarity in behavior between the initial spikes and the late activities has been also discussed by them, based on the functional separation of those components which can be accomplished by applying various parameters of vestibular stimulation, vestibular conditioning and test shocks, etc. It is likely that the initial spike is due to activation of the ventral horn cells which are monosynaptically connected with the vestibular nucleus, but that the late activities are evoked by impulses conducted through poly-synaptic channels with the first synapses residing within the bulb.

It is also evident, as reported ${ }^{1,4,5,7,10}$ earlier that Deiters nucleus stimulation activates both alpha and gamma motoneurons in the ventral horn cells and then affects muscle spindle activity. This implies the strong influence of vestibular function on the maintenance of posture and the orientation in space. The present experiment revealed that the response to vestibular stimulation was modulated by cervical sympathetic stimulation. It is suggested that irritation of the cervical sympathetic nerves caused by neck injury possibly affects the vestibular function which may play a role on maintenance of the neck posture, and that fluctuation of the head position would then result. Thus, injury of the cervical sympathetic nerve can be pointed out as one of the etiological factors producing vertigo.

The potential evoked by sympathetic stimulation recorded from the radial nerve seems to be produced by a mechanism which involves a viscero-somatic reflex arc. The visceromotor reflex has been clearly analysed by Downman. ${ }^{8}$ He concluded that this reflex was mediated by afferent pathways consisting of the A gamma-delta fibers in the sympathetic chain. Excitation of this afferent system elicits skeletal muscle responses multisynaptically. The response recorded from the radial nerve by sympathetic stimulation is similar in latency and shape as well as in behavior to those obtained with repetitive stimulation as reported by Downman. ${ }^{8}$

There is a possibility that adrenal secretion caused by sympathetic stimulation might affect the vestibular responses. The effect of adrenaline injection on the response was examined in this experiment, but no effect was recognized. Anatomic examination also supports existence of centrifugal neurons in the sympathetic ganglia (Fig. 8). These findings favor the view that the inhibitory effect, especially on their late activities, is mediated 
A

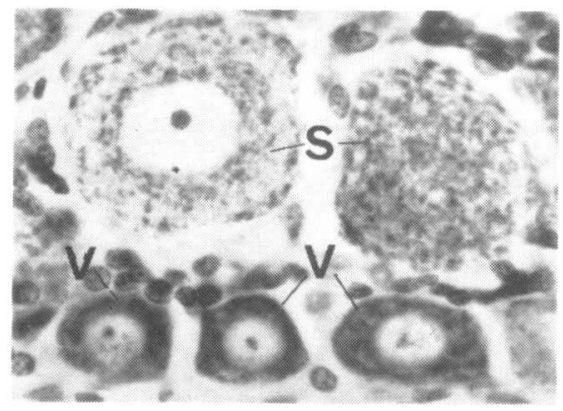

B

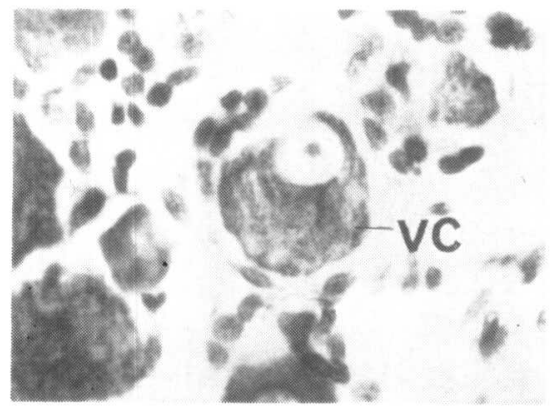

Fig. 8. Microphotographs of the cervical dorsal ganglion. Larger somatic (S) and smaller visceral $(V)$ afferent neurons are seen in $A$. B presents retrograde degeneration of the visceral neuron (VC) following transection of the cervical sympathetic chain between superior and inferior ganglia.

by afferent systems involved in the sympathetic chain.

Stimulation of the cervical dorsal root produced marked inhibition of the late activity. It indicates that injury of the cervical dorsal root caused by whiplash injury could be one of the dominant factors producing vertigo. It has been reported by Wilson, Kato, Thomas \& Peterson ${ }^{13}$ that facilitation of Deiters cell activity was obtained when single shocks were applied on muscle nerves as well as cutaneous or mixed nerves, though the former was less effective than the latter, and no inhibition by peripheral afferent stimulation was obtained. This difference seems to depend on parameters of the conditioning stimulus, since repetitive pulses were used in our experiment. It is also suggested that inhibition of the late activity produced by dorsal root stimulation is exerted at a level of the final common path.

The reversed effect of decerebellation on the influence of sympathetic stimulation on the recovery curves shows that inhibition of the late activity mediated by the sympathetic or visceral afferent nerves would occur at a higher level than that produced by the somatic afferent nerves, at least via the cerebellum. A cortical representation of the splanchnic nerve on the orbital cortex has been recently demonstrated by Korn. ${ }^{12} \mathrm{He}$ suggests that this area is involved in the control of viscero-somatic reflexes. Cangiano, Cook \& Pompeiano ${ }^{3}$ postulated that the cerebellum exerts a differential inhibitory effect on the lateral (Deiters) and medial vestibular nuclei. It seems to be possible that the cerebellum is also involved in this control mechanism. Anyhow, it is interesting that the vestibular nucleus is functionally related to not only the somatic but the visceral afferent systems.

More recently, Barnes \& Pompeiano ${ }^{2}$ have reported that Deiters and the medial vestibular nuclei elicit antagnoistic effects on the monosynaptic reflex pathway in the lumbar cord. In this experiment, however, only Deiters nucleus was examined to analyse vestibular function. The possible contribution of other nuclei and neuronal networks within the vestibular nuclei were not ruled out by this experiment.

Cobbold, Megirian \& Sherrey ${ }^{6}$ having reported that vestibular stimulation evoked 
discharges in the vagus and sympathetic motor outflow, functional relation between the vestibular nucleus, one of the nuclei in the extra-pyramidal systems, and the autonomic nervous system should be analysed further.

\section{SUMMARY}

Effects of cervical dorsal root and sympathetic stimulations on the vestibular motoneurons responses recorded from the radial nerve of the cat were analysed to calrify etiological factors involved in vertigo caused by whiplash injury.

The late activities of the vestibular response were attenuated during repetitive stimulation of the cervical sympathetic nerve. These activities were also inhibited by cervical dorsal root stimulation. No effect was seen in the initial spike of the vestibular response during such modulating stimulation.

However, the inhibitory effect of sympathetic stimulation on the initial spikes was detected when the recovery curve of the initial spike during sympathetic stimulation was compared with that during a no stimulation period. This comparison of the two recovery curves also revealed inhibitory effects on the late activities.

Based on these results, some etiological factors in vertigo as a sequelae of whiplash injury were discussed.

\section{REFERENCES}

1) Anderson, S. \& Gernandt, B. E. : Ventral root discharge in response to vestibular and proprioceptive stimulation. J. Neurophysiol. $19: 524-543,1956$.

2) Barnes, C. D. \& Pompeiano, O. : Dissociation of presynaptic and postsynaptic effects produced in the lumbar cord by vestibular volleys. Arch. ital. Biol. $108: 295-324,1970$.

3) Cangiano, A., Cook, W. A. Jr. \& Pompeiano, O. : Cerebellar inhibitory control of the vestibular reflex pathways to primary afferents. Arch. ital. Biol. $107: 341-364,1970$.

4) Carli, G., Diete-Spiff, K. \& Pompeiano, O.: Responses of the muscle spindles and of the extrafusal fibres in an extensor muscle to stimulation of the lateral vestibular nucleus in the cat. Arch. ital. Biol. $105: 209-242,1967$.

5) Carli, G., Diete-Spiff, K. \& Pompeiano, O.: Mechanism of muscle spindle excitation. Arch. ital. Biol. $105: 273-289,1967$.

6) Cobbold, A. F., Megirian, D. \& Sherrey, J. H. : Vestibular evoked activity in autonomic motor outflows. Arch. ital. Biol. $106: 113-123,1968$.

7) Diete-Siff, K., Carli, G. \& Pompeiano, O. : Comparison of the effects of stimulation of the VIIIth cranial nerve, the vestibular nuclei or the reticular formation on the gsatrocnemius muscle and its spindles. Arch. ital. Biol. $105: 243-272,1967$.

8) Downman, C. B. B. : Skeletal muscle reflexes of splanchnic and intercostal nerve origin in acute spinal and decerebrate cats. J. Neurophysiol. $18: 217-235,1955$.

9) Gernandt, B. E., Katsuki, Y. \& Livingston, R. B. : Functional organization of descending vestibular influences. J. Neurophysiol, $20: 453-469,1957$.

10) Gernandt, B. E., Iranyi, M. \& Livingston, R, B. : Vestibular influences on spinal mechanisms, Exp. Neur. 1 : 248-273, 1959. 
11) Gernandt, B. E. \& Gilman, S. : Descending vestibular activity and its modulation by proprioceptive, cerebellar and reticular influences. Exp. Neur. 1 : 274-304, 1959.

12) KorN, H. : Splanchnic projection to the orbital cortex of the cat. Brain Research $16: 23-38$, 1969.

13) Wilson, V. J., Kato, M., Thomas, R. C. \& Peterson, R. W. : Excitation of lateral vestibular neurones by peripheral afferent fibers, J. Neurophysiol., 29, 508-529, 1966.

(Received March 1, 1971) 